\title{
The Path through sPHENIX and fsPHENIX Toward an EIC Detector at eRHIC
}

\author{
Klaus Dehmelt*† \\ Stony Brook University, Stony Brook, NY 11794-3800, USA \\ E-mail: klaus.dehmeltestonybrook.edu
}

eRHIC is one of the options proposed as a high luminosity, polarized Electron-Ion Collider (EIC) that is based on using one of the RHIC hadron rings and a multipass Energy Recovery Linac. A beam of polarized electrons with an energy up to $21 \mathrm{GeV}$ would collide with a variety of ion species, from polarized protons with a top energy of $250 \mathrm{GeV}$ to fully-stripped uranium ions with energies up to $100 \mathrm{GeV} / \mathrm{u}$. It will allow coverage of a center-of-mass energy range up to 126 $\mathrm{GeV}$ for polarized $\mathrm{e}+\mathrm{p}$, and up to $80 \mathrm{GeV}$ for e+A (for large $\mathrm{A}$ ) collisions. One of the possible detectors to perform precision studies of the partonic structure of hadronic matter will be an upgraded PHENIX detector. The path will lead through the detector that will be built around the Babar magnet, sPHENIX, with its components at midrapidity $|\eta|<1$ followed by an sPHENIXForward upgrade and additional modifications specific to the successive EIC detector. We will discuss the approach that leads to that EIC detector and present goals and requirements for a broad set of measurements from Day-1 of eRHIC.

XXIV International Workshop on Deep-Inelastic Scattering and Related Subjects

11-15 April, 2016

DESY Hamburg, Germany

\footnotetext{
${ }^{*}$ Speaker.

${ }^{\dagger}$ For the PHENIX collaboration.
} 


\section{Introduction}

The U.S. Nuclear Science community is considering a high energy, high luminosity, polarized proton-electron and light nuclei-electron, respectively ion-electron collider (EIC) as its next large experimental facility [1]. Two possible scenarios for the location of the EIC are presently being discussed. One location is considered to be at the BNL-RHIC facility which is planning to add an electron accelerator with energies up to $21 \mathrm{GeV}$ (eRHIC). The other location would be at JLabCEBAF which is planning to add a beam facility that could accelerate $20-100 \mathrm{GeV}$ protons or up to $40 \mathrm{GeV} / \mathrm{u}$ ions (MEIC).

\section{2. eRHIC}

The eRHIC design is based on using one of the two existing hadron rings from RHIC and adding a multipass energy recovery linac (ERL) and offers the possibility of more than one interaction region (Fig. 1) in one of which it is proposed to use the planned sPHENIX detector with appropriate modifications/upgrades to become an EIC-detector from Day-1 [2]. The existing RHIC accelerator would provide polarized protons up to $\mathrm{E}=250 \mathrm{GeV}$ and fully stripped uranium ions up to $100 \mathrm{GeV} / \mathrm{u}$. The ERL would provide polarized electrons up to $\mathrm{E}=16 \mathrm{GeV}(21 \mathrm{GeV})$ corre-

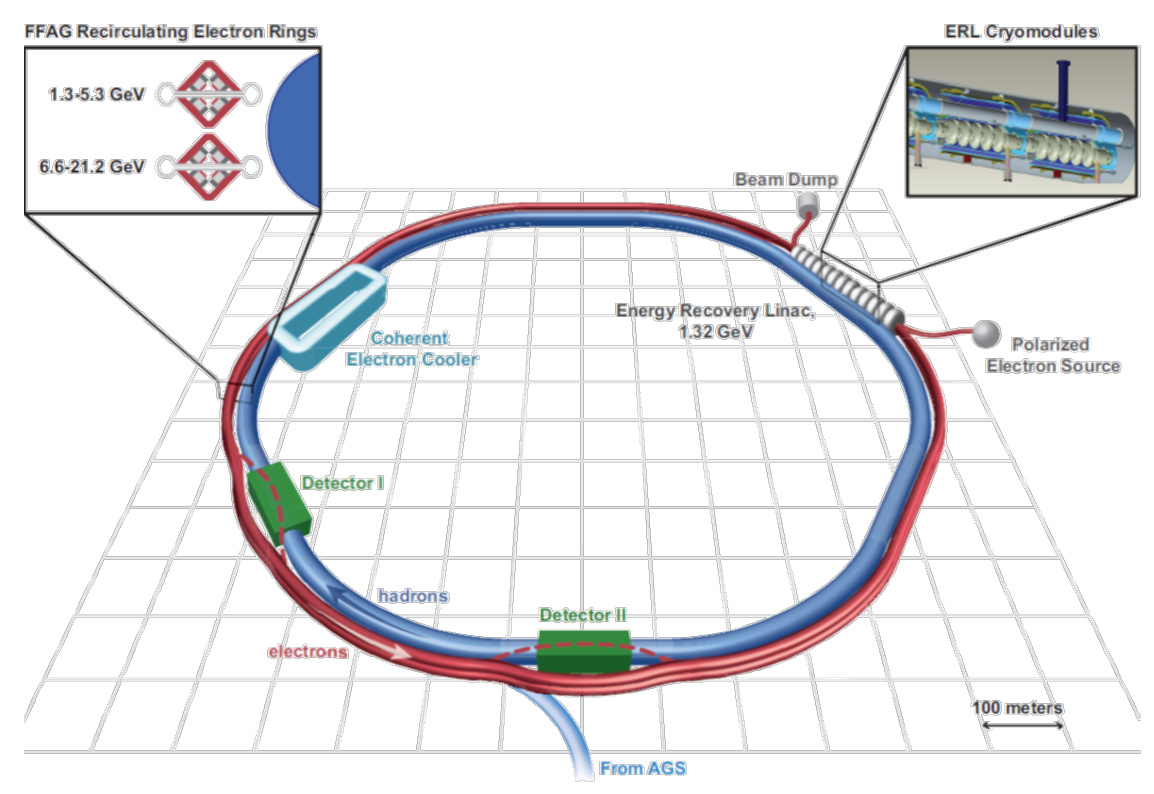

Figure 1: eRHIC design with one of the two hadron rings from RHIC being used as hadron accelerator and a newly built ERL, with two interaction regions.

sponding up to $\sqrt{s}=126 \mathrm{GeV}(145 \mathrm{GeV})$ for polarized e-p collisions and up to $\sqrt{s}=80 \mathrm{GeV}$ (91 $\mathrm{GeV}$ ) for e-A collisions with large A. It is planned to achieve luminosities of $10^{33} \mathrm{~cm}^{-2} \mathrm{~s}^{-1} \leq \mathscr{L} \leq$ $10^{34} \mathrm{~cm}^{-2} \mathrm{~s}^{-1}$ whereas it is expected that the luminosity will drop by a factor two or three for the case of highest $\sqrt{s}$.

The target is to provide an eRHIC facility from Day-1 with one high-luminosity intersection region that can take advantage of the existing infrastructure in the STAR and PHENIX experimental halls. 
A Day-1 eRHIC detector is planned to be the successor of the sPHENIX experiment that will be built around the BaBar solenoid.

\section{Physics Deliverables}

The Day-1 eRHIC detector [3] has set forth deliverables to accomplish the physics program: the investigation of the longitudinal spin of the proton, the transverse motion of quarks and gluons in proton, performing tomographic imaging of the proton, investigating hadronization and its modification in nuclear matter, as well as QCD matter at extreme gluon density. The spin program
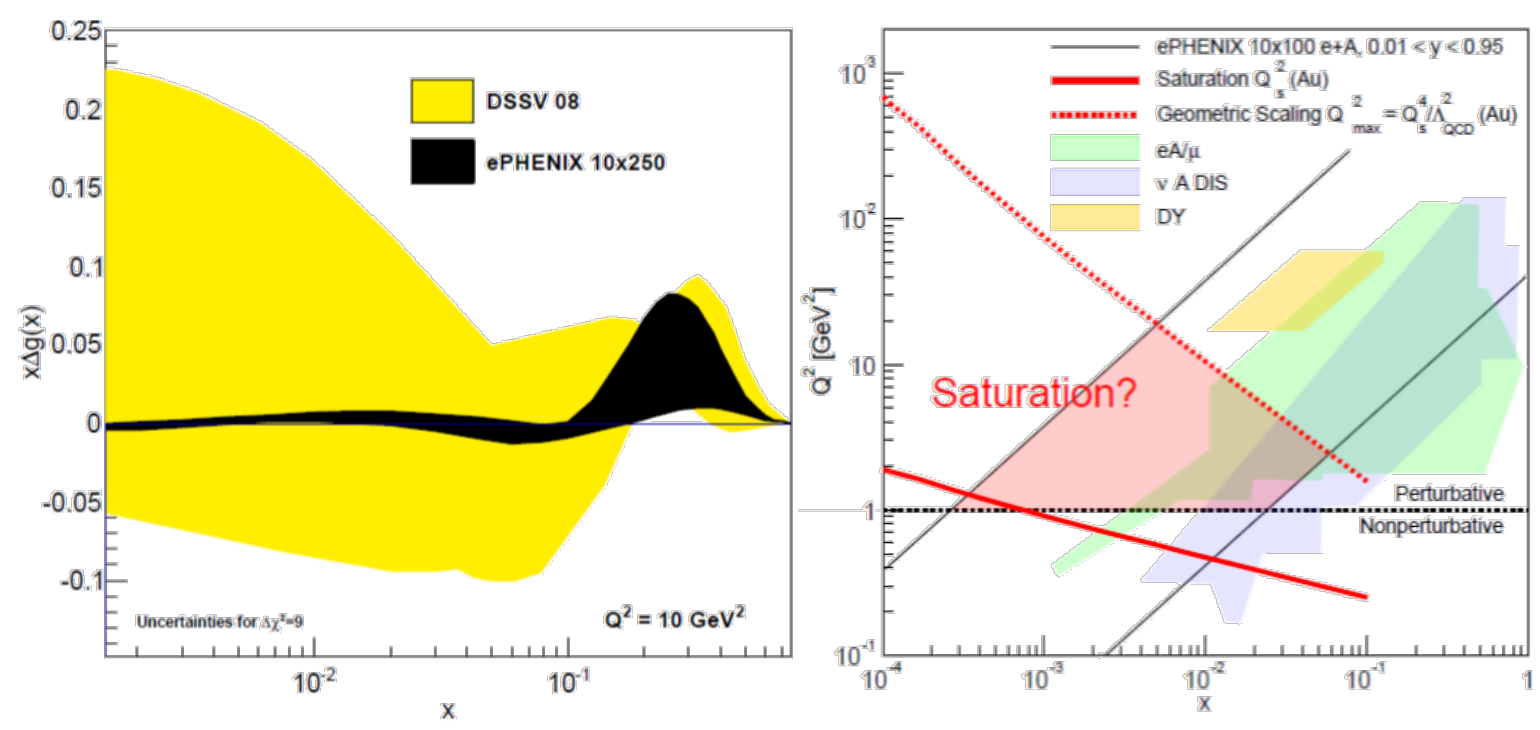

Figure 2: Left: Projected reduction in the uncertainty (black) on the gluon longitudinal spin distribution based on simulated PYTHIA events at the $10 \mathrm{GeV} \times 250 \mathrm{GeV}$ beam energy configuration. Right: Shown is the coverage in $\mathrm{x}$ and $\mathrm{Q}^{2}$ for the EIC and the ePHENIX detector for $10 \mathrm{GeV}$ electrons on $100 \mathrm{GeV} /$ nucleon heavy nuclei.

is aiming to reduce the uncertainty on gluon longitudinal spin distribution (Fig. 2, left); transverse motion can be investigated by looking into the transverse momentum of, e.g., the up-quark; investigating QCD matter at extreme gluon density can be accomplished by probing saturation effects (Fig. 2, right), to name a few. The Day-1 eRHIC detector design will provide coverage of a large region in $\mathrm{X}^{-} \mathrm{Q}^{2}$-space (Fig. 3).

\section{Detector Requirements}

The physics program of an eRHIC detector has stringent requirements on sub-detectors, of which many are beyond state-of-the-art. Electron identification (ID) needs electromagnetic calorimetry and charged particle tracking with a minimum material budget before EMCal and good energy and tracking resolution for E/p matching. Hadron ID can be performed with DIRC technology in the acceptance region of the barrel, for hadron momenta $\mathrm{p}_{h}<4 \mathrm{GeV} / \mathrm{c}$; in the hadron-going direction Aerogel-technology can be used for lower momentum and the gas RICH for higher momenta. Electron/Photon separation can be achieved with high granularity EMCal in the the electron-going 


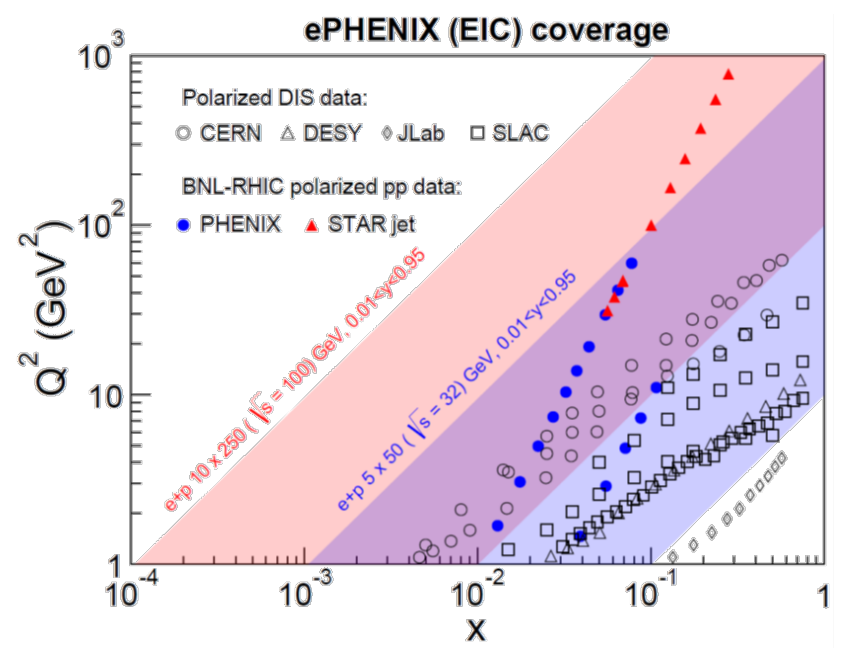

Figure 3: Kinematic coverage of ePHENIX for two beam energy configurations, $10 \tilde{A} U \circ 250 \mathrm{GeV}$ and 5 ÃŮ50 $\mathrm{GeV}$, which show the range of eRHIC capabilities.

direction.

Resolution in $\mathrm{x}$ and $\mathrm{Q}^{2}$ can be obtained by means of a high resolution EMCal and tracking in electron-going direction. Good momentum resolution, i.e., tracking is required for $\mathrm{E}_{e}^{\prime}<10 \mathrm{GeV}$ (scattered electron energy) in the barrel acceptance. Good energy, i.e., EMCal resolution is needed for $\mathrm{E}_{e}^{\prime}>10 \mathrm{GeV}$ in the barrel acceptance.

Wide acceptance for Leptons and Photons in DVCS requires EMCal and tracking with good resolution for lepton and photon measurements covering $|\eta|<4$. Scattered protons in exclusive processes can be measured with Roman pots in the hadron-going direction.

Measuring the rapidity gap requires hadronic calorimetry covering $-1<\eta<5$ and EMCal covering $|\eta|<4$. A Forward Zero-Degree calorimeter in the hadron-going direction is planned, in coordination with Collider Accelerator Department CAD.

\section{A Day-1 eRHIC Detector}

The sPHENIX detector [4] will consist of hadronic and electromagnetic calorimeters, built around the Babar Magnet. It will be completed with a barrel tracking system and forward electromagnetic calorimeters (Fig. 4, left).

In the era of a possible forward physics program it is desired to complement the existing sPHENIX detector with forward tracking and hadronic calorimeter capabilities and will be receiving the name fsPHENIX. This detector will be used as basis and by adding forward and barrel particle ID components turn into a Day-1 eRHIC detector capable to take on the physics program mentioned in the previous sections.

\section{Performance of a Day-1 eRHIC Detector}

The tracking performance of a Day-1 eRHIC detector is shown in Fig. 5, left for the various pseudorapidity regions which ranges from $-3<\eta<4$. For the case of using the $20 \mathrm{GeV}$ electron 


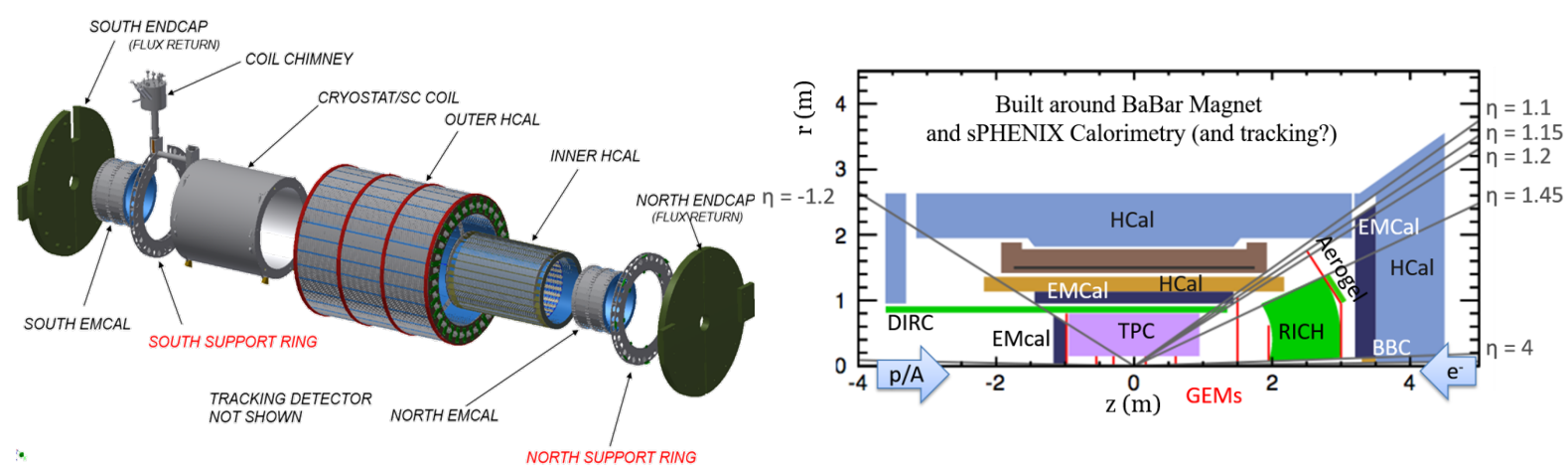

Figure 4: Left: Exploded view of the sPHENIX detector. Right: Sideview of a Day-1 eRHIC detector.

beam one needs to go down as far as $\eta=-4$, however, this will only be a minor reduction in the $x-Q^{2}$-space as can be seen in Fig. 5, right. For measuring quark helicity, TMD and hadronization,
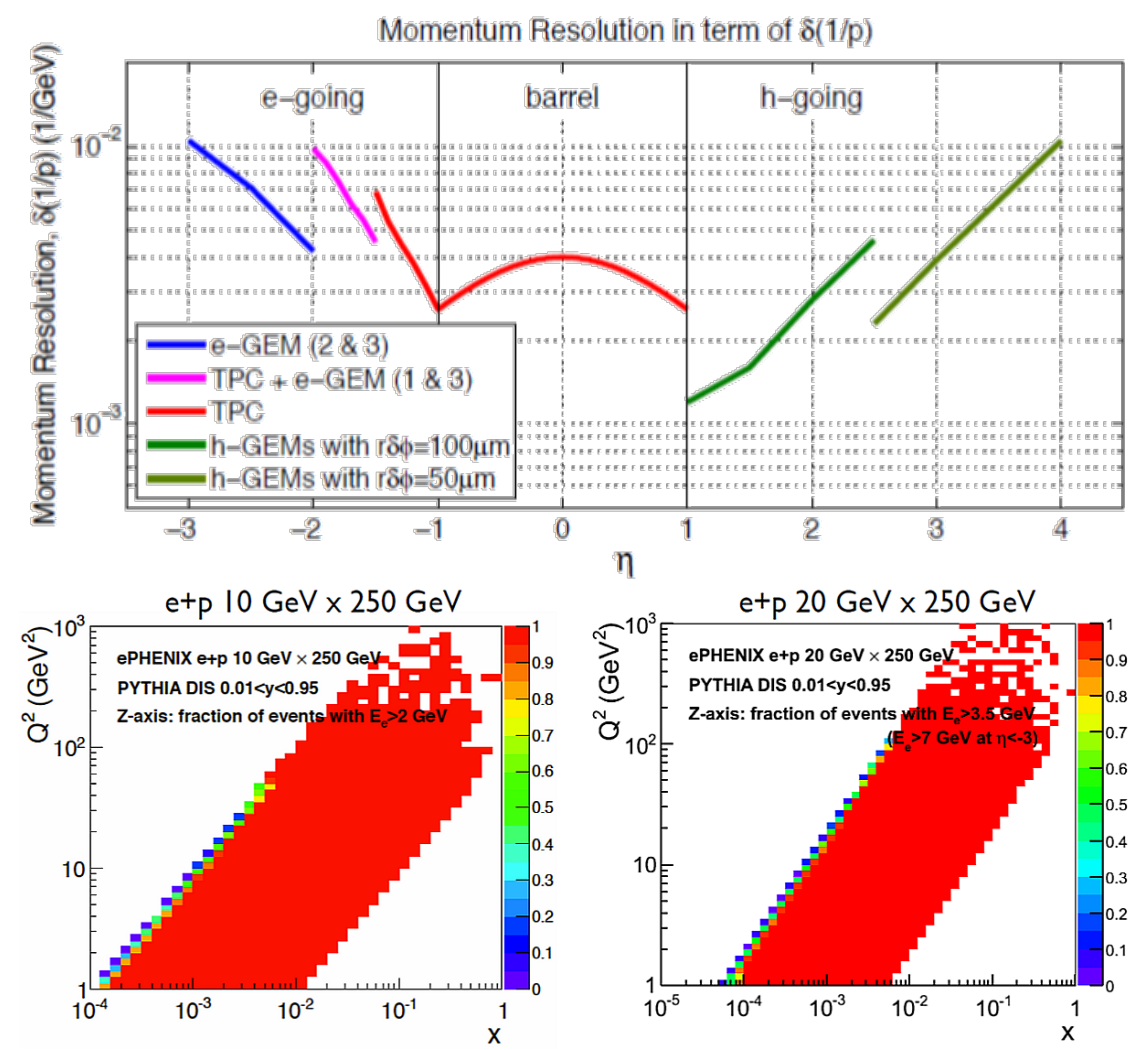

Figure 5: Upper: Tracking performance of a Day-1 eRHIC detector within various $\eta$-regions. Lower: Comparison in $\mathrm{x}-\mathrm{Q}^{2}$-space for $\mathrm{e}+\mathrm{p}$ collisions at $10 \mathrm{GeV} \times 250 \mathrm{GeV}$ respectively $20 \mathrm{GeV} \times 250 \mathrm{GeV}$.

high demands are set for identifying kaons in the forward hadron-going direction. $\pi$-K separation as far as to $70 \mathrm{GeV} / \mathrm{c}$ and down to $\eta=4$ is needed which will be accomplished with the forward PID capability of a Day-1 eRHIC detector (Fig. 6). The performance of a Day-1 eRHIC detector here shown is an excerpt and is more thoroughly discussed in [3]. 

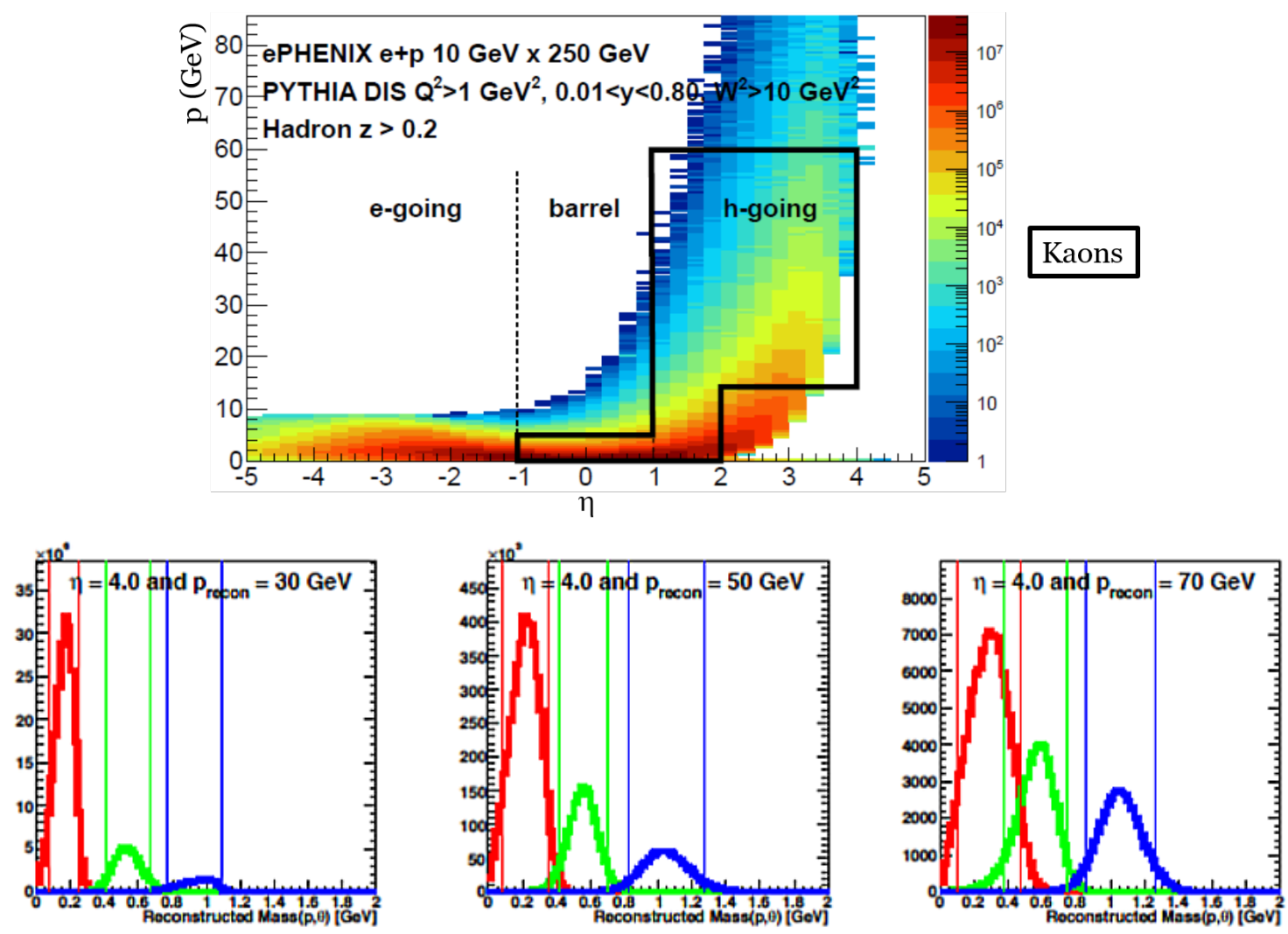

Figure 6: Upper: Distribution of hadrons (Kaons) from DIS events in $\mathrm{e}+\mathrm{p}$ as a function of momentum and pseudorapidity Lower: $\pi / \mathrm{K} / \mathrm{p}$ separation at various reconstructed lab momenta.

\section{Summary}

Many opportunities are available to study condensed matter of the strong force from Day-1. A path from the sPHENIX detector, with its focus on jets and hard probes in heavy-ion collisions, into a eRHIC detector seems to be promising. Additional capabilities supporting its focus on $\mathrm{e}+\mathrm{p}$ and e+A collisions the sPHENIX detector in the existing PHENIX experimental hall is well capable to be used as a Day-1 eRHIC detector. It will make full use of PHENIX upgrades to sPHENIX and Forward sPHENIX with additional specific modifications for EIC physics.

\section{References}

[1] Electron Ion Collider: The Next QCD Frontier - Understanding the glue that binds us all, arXiv:1212.1701

[2] eRHIC Design Study: An Electron-Ion Collider at BNL, arXiv:1409.1633

[3] Concept for an Electron Ion Collider (EIC) detector built around the BaBar solenoid, arXiv:1402.1209

[4] sPHENIX preConceptual Design Report, https://indico.bnl.gov/getFile.py/access?resId=0\&materialId=11\&confId=1483 